\title{
Neurosarcoidosis: Granulomatous Disease Presenting with Confusion and Gait Disturbance
}

\author{
Jasmine Mitchell, MS, Divya Chalikonda, MD, and Drew Kotler, MD
}

\section{INTRODUCTION}

Sarcoidosis is a multisystem disorder characterized by noncaseating granulomas that frequently presents with pulmonary infiltrates, hilar lymphadenopathy, and ocular and skin lesions. Sarcoidosis affects about 20 per 100,000 individuals in the US with a higher prevalence among African Americans than in Caucasians. ${ }^{1}$ The average age of presentation ranges from 33 to 41 years. Less than $10 \%$ of people with sarcoidosis have CNS involvement, with cranial neuropathy, aseptic meningitis, hydrocephalus, seizures, psychiatric symptoms, and cerebral lesions with endocrine manifestations included among the possible manifestations. ${ }^{2}$ This case report describes a woman with neurosarcoidosis presenting with confusion and gait disturbances.

\section{CASE PRESENTATION}

A 59-year-old woman with a past medical history of hypertension and diabetes presented with one month of progressive confusion, lower extremity weakness, and gait instability. Prior to admission, she was treated at an outside hospital for presumed aseptic meningitis without improvement. The following diagnoses were present on admission: acute kidney injury with a creatinine of 2.1 $\mathrm{mg} / \mathrm{dL}$, a FeNa of $3.3 \%$, and hypercalcemia to $14.5 \mathrm{mg} /$ $\mathrm{dL}$. The physical exam was only notable for a persistent low grade fever averaging $100.3^{\circ} \mathrm{F}$. She had no other symptoms.

During the course of her admission, she developed polyuria which prompted a discussion of possible diabetes insipidus. A brain MRI without contrast ordered to evaluate this possibility showed mild dilatation of the lateral and third ventricles, suggestive of non-obstructive hydrocephalus. A subsequent lumbar puncture revealed an elevated opening pressure and elevated protein of 64 $\mathrm{mg} / \mathrm{dL}$. In the CSF, the ACE level was normal at $8 \mathrm{U} / \mathrm{L}$, the glucose level was $51 \mathrm{mg} / \mathrm{dL}$, and oligoclonal bands were seen. No other cytological or pathological findings were noted.

Given the lack of pathology within the CNS, a CT of the chest, abdomen, and pelvis was done and demonstrated bilateral pulmonary nodules and diffuse mediastinal, supraclavicular, and intra-abdominal lymphadenopathy (Figure 1). Suspicious for lymphoma, an axillary lymph node biopsy was performed. Pathology showed non-necrotizing granulomatous inflammation (Figure 2), consistent with sarcoidosis. Flow cytometry showed normal cytology and cultures were negative. Corticosteroids were empirically started while further infectious causes were ruled out. Within a week, her symptoms improved significantly with improved gait and cleared sensorium. Her polyuria, likely from central diabetes insipidus secondary to neurosarcoidosis, also improved.

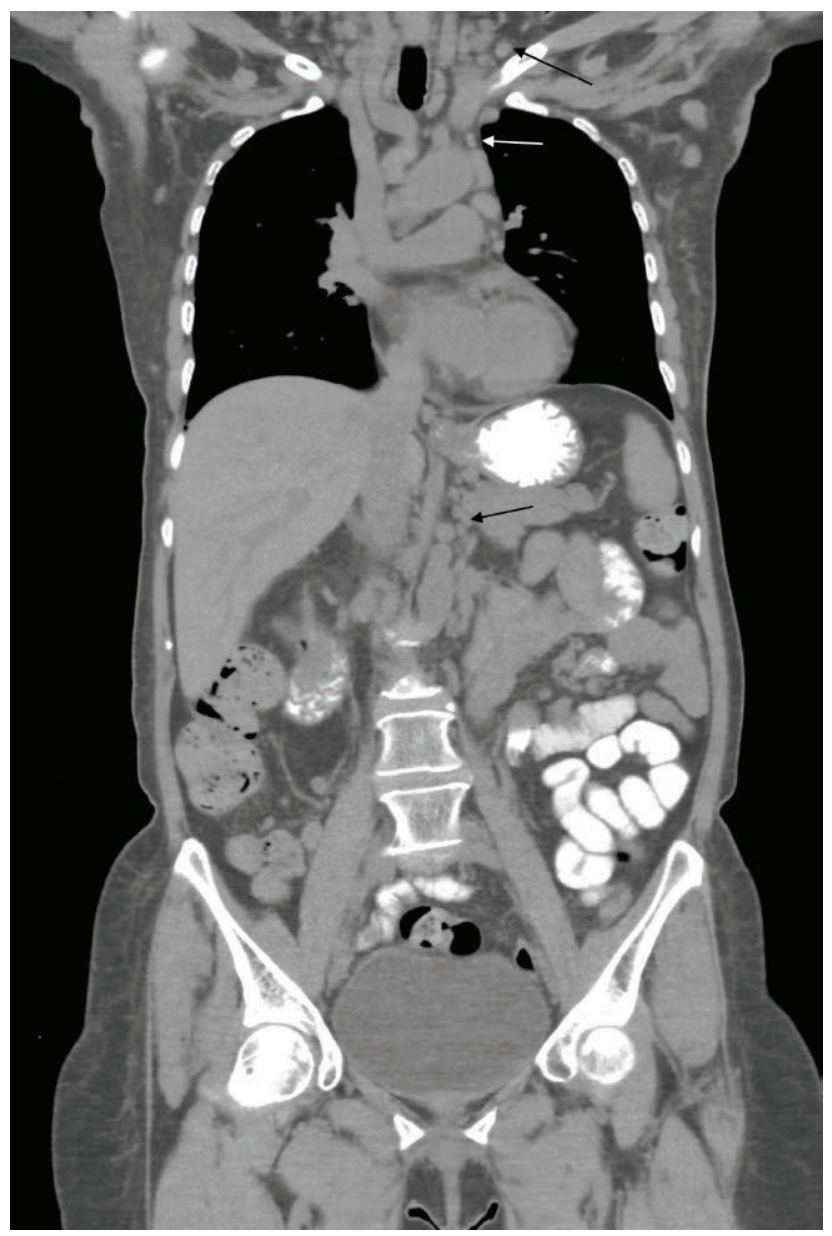

Figure 1. CT of the chest, abdomen, and pelvis demonstrating diffuse lymphadenopathy. 


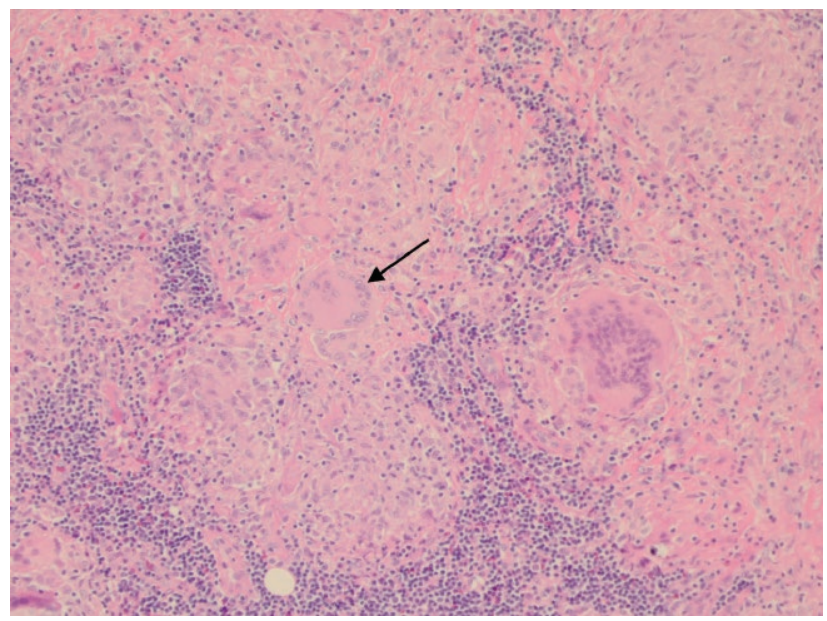

Figure 2. Noncaseating granulomas seen on the lymph node biopsy (arrow points to a multinucleated giant cell)

\section{DISCUSSION}

Diagnosis of neurosarcoidosis is difficult as it can resemble other infectious diseases, neoplasms, and demyelinating diseases. As mentioned, infectious causes such as histoplasmosis, toxoplasmosis, tuberculosis, and JC virus were all ruled out. Clinically, multiple sclerosis can also resemble neurosarcoidosis, and in this case, oligoclonal bands were seen in the CSF which raises suspicion for MS. However, oligoclonal bands are observed in $19-37 \%$ of neurosarcoidosis cases. ${ }^{3}$ The patient's lack of a relapsing and remitting course combined with the presence of hydrocephalus, meningitis, and hypothalamic/pituitary involvement favored the diagnosis of neurosarcoidosis over multiple sclerosis

Hypercalcemia can be seen in approximately $10 \%$ of sarcoidosis patients. ${ }^{4}$ This patient's normal calcitriol levels were inconsistent with the elevated calcitriol levels expected in neurosarcoidosis, as excess calcitriol is produced by macrophages and other activated mononuclear cells in the lung and lymph nodes. Despite this negative finding, a lymph node biopsy showed non-caseating granulomas

Biopsy is the gold standard for diagnosis. Brain biopsies account for only $10-30 \%$ of diagnostic biopsies, with lung or lymph node biopsies occurring more frequently. ${ }^{3}$ In this patient's case, histologic confirmation of noncaseating granulomas in addition to the exclusion of other diseases enhanced the likelihood of neurosarcoidosis as the final diagnosis. Once diagnosis is confirmed, corticosteroids are the drug of choice for treatment. In the case of long-term use or refractory sarcoidosis, second-line treatment would include methotrexate and azathioprine. ${ }^{3}$ Third-line treatment includes TNF- $\alpha$ inhibitors. ${ }^{5}$

\section{CONCLUSION}

This is an uncommon presentation of sarcoidosis with CNS involvement as the main concern. The case demonstrates the importance of obtaining a tissue biopsy, especially in the absence of other pathognomonic features of disease. Using appropriate clinical judgment in regards to starting steroids is crucial while non-immunologic etiologies are being ruled out, as prompt treatment will reverse its potentially detrimental sequelae.

\section{REFERENCES}

1. Erdal BS, et al. Unexpectedly high prevalence of sarcoidosis in a representative U.S. Metropolitan population. Respiratory Medicine. 2012;106(6):893-899.

2. Lacomis D. Neurosarcoidosis. Current Neuropharmacology. 2011;9(3):429-436

3. Nozaki K, Judson M. Neurosarcoidosis: Clinical manifestations, diagnosis and treatment. La Presse Medicale. 2012;41:331-348.

4. James D. A clinicopathological classification of granulomatous disorders. Postgrad Med Journal. 2000;76:457-465.

5. Beegle $\mathrm{SH}$, et al. Current and emerging pharmacological treatments for sarcoidosis: a review. Drug Design, Development and Therapy. 2013:7:325-338 